\title{
Synthesis, Characterization and In Vitro Antibacterial Derivatives of Doxycycline
}

\author{
Muhammad Rizwan Khan', Mohsin Abbas Khan', Khalil Ahmad ${ }^{2, *}$, Asad Hamad', Muhammad Sajid-ur-

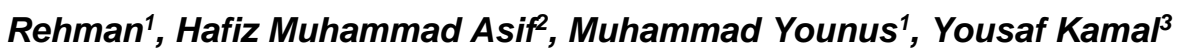 \\ ${ }^{1}$ Department of Pharmacy, The Islamia University of Bahawalpur, Bahawalpur, Pakistan \\ ${ }^{2}$ University College of Conventional Medicine, The Islamia University of Bahawalpur, Bahawalpur, Pakistan \\ ${ }^{3}$ Department of Pharmacy, COMSAT, Abbottabad, Pakistan
}

\author{
Authors' Contributions \\ 1 Conception \& Study Design. \\ 2 Data Collection. \\ 3,6 Data Analysis. \\ 4,5,8 Drafting. \\ 7 Critical Review.
}

\section{Article info.}

Received: January 17, 2019

Accepted: November 13, 2019

Funding Source: Nil

Conflict of Interest: Nil

Cite this article: Khan MR, Khan MA, Ahmad

K, Hamad A, Sajid-ur-Rehman M, Asif HM,

Younus M, Kamal Y. Synthesis,

Characterization and In Vitro Antibacterial

Derivatives of Doxycycline. RADS J Pharm

Pharm Sci. 2019; 7(4): 215-226.

*Address of Correspondence Author: ansari.khalil@gmail.com

\section{ABSTRACT}

Objective: Nowadays antibacterial drugs resistance is major problem in the world. To overcome this problem, some novel derivates of doxycycline were synthesized by single step condensation reaction with ten different types of aromatic and aliphatic aldehydes and ketones in ethanol as solvent and acetic acid as catalyst. In these reactions, deprotonation of primary amine occurs that results in formation of imine.

Methods: All the derivatives physically characterized and confirmed by analytical techniques i.e. FTIR and ${ }^{1} \mathrm{H}$ NMR and ${ }^{13} \mathrm{C}$ NMR. The derived compounds have exhibited significantly more active against both gram positive as well as gram negative bacterial strains as compared to parent drug.

Results: Derived Schiff bases RDC2, RDC4 and RDC10 showed zone of inhibition against Bacillus subtilis as compared to doxycycline and derived Schiff bases RDC1, RDC2, RDC5, RDC6, RDC7 and RDC8 showed more zone of inhibition against Micrococcus luteus as compared to doxycycline. Yield $(75 \%)$, m.p. $180-185^{\circ} \mathrm{C}$, Mol. Wt. 1063 , Elemental Analysis: (Calculated) for $\mathrm{C}_{58} \mathrm{H}_{54} \mathrm{~N}_{4} \mathrm{O}_{16}$ : ' $\mathrm{C}, 65.53 ; \mathrm{H}, 5.12 ; \mathrm{N}, 5.27$; (Found): C, 65.49; H, 5.18; N, 5.37; FTIR $\left(\mathrm{v}, \mathrm{cm}^{-1}\right): 3066(=\mathrm{C}-\mathrm{H}), 1665(\mathrm{C}=\mathrm{N}), 1585,1490(\mathrm{C}=\mathrm{C}$, phenyl), 3650(OH), $1692(\mathrm{C}=\mathrm{O})$; ${ }^{1} \mathrm{H}$ NMR (DMSO-d6, $\left.\delta, \mathrm{ppm}\right), 6.02-6.03 \mathrm{~d}, 6.91-6.92$ $\mathrm{d},(=\mathrm{CH}-) ; 6.88-6.89 \mathrm{t}(=\mathrm{CH}), 2.89-2.90 \mathrm{~d},(-\mathrm{CH}) ; 1.07-1.08 \mathrm{q},\left(-\mathrm{CH}_{3}\right), 3.38-$ $3.39 \mathrm{~d}$; 3.17-3.18 d, $(\mathrm{CH}), 1.47-1.48 \mathrm{t}(\mathrm{CH}), 1.48 \mathrm{~s},\left(\mathrm{CH}_{3}\right), 6.96-6.97 \mathrm{~d}, 7.577-$ $7.588 \mathrm{~d},(-\mathrm{CH}=) 15.21 \mathrm{~s}(\mathrm{OH}) ; 4.62 \mathrm{~s}(\mathrm{OH}) ; 1.46-1.47 \mathrm{~s}(\mathrm{OH}) ;{ }^{13} \mathrm{C} \mathrm{NMR}$ (DMSO-d6, $\delta$, ppm).

Conclusion: Doxycycline is among broad-spectrum tetracycline. The Schiff bases derived from doxycycline show significantly highly active against gram negative bacteria as compared to doxycycline. In future further study on these derived compounds will help in market new derivative of doxycycline, which will have more broad-spectrum activity than doxycycline.

Keywords: Doxycycline, Schiff's bases, FTIR, ${ }^{1} \mathrm{H}$ NMR and ${ }^{13} \mathrm{C}$ NMR. 


\section{INTRODUCTION}

Hugo Schiff in 1864 first time made Schiff base. Schiff bases have functional group containing carbon nitrogen double bond $\left(R_{1} R_{2} C=N R_{3}\right)$. Here $R_{3}$ may be alkyl or aryl group but not hydrogen atom, $R_{1}$ and $R_{2}$ is organic side chain [1].

In 1864 Hugo Schiff made first Schiff base by reaction of aniline with benzaldehyde, acetaldehyde, valeraldehyde and cinnamaldehyde [2]. He opened the new door of synthetic chemistry by synthesis of imine [3]. First beta lactam is produced by reaction of Schiff base with diphenyl ketenes [4].

Aryl substituent containing Schiff bases are more stable then alkyl substituent [5]. Aldehydes react rapidly with imine than ketones because aldehydes have less stearic hindrance than ketones. The second carbon attached with carbonyl group make ketones less electrophilic than aldehydes [6]. The condensation products of aromatic amines and aldehydes and ketones are known as azomethines imines or anils. The aldehydes and ketone derived Schiff bases are known as aldimines and ketamine, respectively. The $\mathrm{C}=\mathrm{N}$ of imines have wide biological and chemical importance. The $\mathrm{C}=\mathrm{N}$ have excellent chelating properties the presence of hydroxyl and thiol group with azomethine group may form penta or hexa ring structure with metal ions. Schiff bases may be bidentate, tridentate, tetradentate, and polydentate. Aliphatic aldehydes derived Schiff bases less stable than aromatic aldehydes because conjugation make them more stable.

Schiff bases have interesting biological properties because of electron donating and electron accepting groups [7]. There is wide range of biological activity of Schiff bases i.e. antimicrobial activity against bacteria protozoa fungal and viral infection [8] also have anticancer activity [9] anti-inflammatory and analgesic activity [10] and anticonvulsant activity [11] antianthelmintic activity [12]. The carbon nitrogen double bond is mainly responsible for chelating activity of Schiff bases [13]. Schiff bases have important chemical properties these are precursor four different reactions organometallic reactions carbon nitrogen double bond have capability of incorporation of metals [14], in coordination chemistry Schiff bases have unique application due to its chelating property [15]. These metal complexes have antifungal antibacterial anti-cancer activities [16, 17].

\section{Doxycycline}

Pfizer chemists team led by Robert Blackwood first time synthesized doxycycline from methacycline and it was approved 1967 by FDA and marketed as vibramycin [18]. It is one of the drug in WHO essential drugs list most effective and safe medicine among antibiotics for human body system [19]. The mechanism of action of doxycycline "it inhibits microorganism macromolecule synthesis by binding to the 305 microorganism organelle and preventing access of aminoacyl soluble RNA to the acceptor site on the mRNA-ribosome advanced. These medications enter gram-negative microorganism by passive diffusion through the hydrophilic channels fashioned by the porin proteins of the outer plasma membrane associated by transport via an energydependent system that pumps all doxycycline across the living substance membrane. Entry of those medication into gram-positive microorganism needs metabolic energy, however isn't in addition understood" [20].

It is use in acute pelvic inflammatory disease with combination of cefoxitin [21] in ocular rosacea [22].

\section{MATERIALS AND METHODS}

\section{List of Chemicals}

All the chemicals used were analytical grade and obtained from the following sources (Table 1):

Table 1. List of chemicals used.

\begin{tabular}{|c|c|c|}
\hline Chemical & Formula & Supplier \\
\hline Ethanol & $\mathrm{C}_{2} \mathrm{H}_{5} \mathrm{OH}$ & Sigma \\
\hline Benzil & $\mathrm{C}_{14} \mathrm{H}_{10} \mathrm{O}_{2}$ & Sigma \\
\hline Salicylaldehyde & $\mathrm{C}_{7} \mathrm{H}_{6} \mathrm{O}_{2}$ & Sigma \\
\hline Cinnamaldehyde & $\mathrm{C}_{9} \mathrm{H}_{8} \mathrm{O}$ & Sigma \\
\hline Vanillin & $\mathrm{C}_{8} \mathrm{H}_{8} \mathrm{O}_{3}$ & Sigma \\
\hline Acetone & $\mathrm{C}_{3} \mathrm{H}_{6} \mathrm{O}$ & Merck \\
\hline Acetophenone & $\mathrm{C}_{8} \mathrm{H}_{8} \mathrm{O}$ & Merck \\
\hline Benzaldehyde & $\mathrm{C}_{7} \mathrm{H}_{6} \mathrm{O}$ & Sigma \\
\hline Benzophenone & $\mathrm{C}_{13} \mathrm{H}_{10} \mathrm{O}$ & Merck \\
\hline Formalin & $\mathrm{HCOH}_{1} \mathrm{COH}_{14}$ & Merck \\
\hline Benzoin & $\mathrm{C}_{14} \mathrm{H}_{12} \mathrm{O}_{2}$ & Merck \\
\hline Acetic Acid & $\mathrm{CH}_{3} \mathrm{COOH}$ & Merck \\
\hline Doxycycline & $\mathrm{C}_{22} \mathrm{H}_{24} \mathrm{~N}_{2} \mathrm{O}_{8}$ & Sigma \\
\hline
\end{tabular}

Doxycycline was obtained from Remington Laboratories Pvt. Ltd. Lahore; its percentage purity was 95. 


\section{Preparation of Schiff Bases}

In $250 \mathrm{ml}$ round bottom flask equimolar solution of available aldehydes and ketones and doxycycline in $30 \mathrm{ml}$ of ethanol added in flask. Few drops of acetic acid also added as catalyst. The reaction mixture refluxed for 4 hours, cool at room temperature and filter. The solvent was evaporated by rotary evaporator the solid residue collected and dried at room temperature. Recrystallize with ethanol. Characterized by using different physio-chemical procedure.

\section{Instrumentation and Spectral Determination}

All the physical properties of prepared Schiff base drugs like solubility and melting points were determined.

Solubility of all the derived ligands were determined by using Ela E 30H, D78224 Singe / Htw sonicator.

Galen Kemp apparatus used to determine the melting point of Schiff base drugs.

FTIR spectra of Schiff bases obtained from Brooker TENSOR 27 FTIR spectrophotometer.

${ }^{1} \mathrm{H}$ NMR and ${ }^{13} \mathrm{C}$ NMR spectra of synthesized were obtained by Bruker 400-spectrophotometer.

Antibacterial activity of all synthesized ligand was performed by using agar well diffusion method. Autoclave was used for sterilization of equipment and media used for activity.

\section{Solubility}

Solubility of all derived Schiff bases is determined one by one in different solvents like water, ethanol, DMSO, methanol and acetone using Ela E $30 \mathrm{H}$, D78224 Singe / Htw sonicator.

\section{Melting Point}

Melting point of all synthesized Schiff bases were determined one by one sample by adding small amount of powder of synthesized Schiff base in capillary tube and tube heated on Galen Kemp melting point apparatus.

\section{Infrared Spectrum}

Infrared spectrum of Schiff bases was determined by Bruker TENSOR-27 FTIR spectrophotometer using $\mathrm{KBR}$ pellets in the range of $4000-400 \mathrm{~cm}^{-1}$ at speed of 16 times per sample.

\section{NMR Spectrum}

${ }^{1} \mathrm{H}$ NMR and ${ }^{13} \mathrm{C}$ NMR spectra of synthesized were obtained by Bruker 400-spectrophotometer.

\section{General Scheme}<smiles>CC1c2cccc(O)c2C(=O)C2=C(O)C3(O)C(=O)C(C(N)=O)=C(O)C(N(C)C)C3C(O)C21</smiles><smiles>[R]C(=O)Br</smiles><smiles>[R]C([R])=NC(=O)C1=C(O)C(N(C)C)C2C(O)C3C(=C(O)C2(O)C1=O)C(=O)c1c(O)cccc1C3C</smiles>

(RDC1 to RDC10)

\section{General scheme for synthesis of Schiff bases of} doxycycline

Where,

$\mathrm{D} 1=\mathrm{RCH} 3 \& \mathrm{R}^{1}: \mathrm{H}$

$\mathrm{D} 2=\mathrm{R}: 2-(\mathrm{OH}) \mathrm{C} 6 \mathrm{H} 4 \& \mathrm{R}^{1}: \mathrm{H}$

$\mathrm{D} 3=\mathrm{R}: \mathrm{C} 6 \mathrm{H} 5 \mathrm{CH}=\mathrm{CH} \& \mathrm{R}^{1} \mathrm{H}$

$\mathrm{D} 4=\mathrm{R}: 3-\left(\mathrm{OCH}_{3}\right) 4-(\mathrm{OH}) \mathrm{C} 6 \mathrm{H} 3 \& \mathrm{R}^{1} \mathrm{H}$

$\mathrm{D} 5=\mathrm{R}: \mathrm{CH} 3 \& \mathrm{R}^{1} \mathrm{CH} 3$

D6 $=\mathrm{R}: \mathrm{C}_{6} \mathrm{H}_{5} \& \mathrm{R} 1: \mathrm{CH}_{3}$

$\mathrm{D} 7=\mathrm{R}: \mathrm{C} 6 \mathrm{H} 5 \& \mathrm{R}^{1}: \mathrm{H}$

$\mathrm{D} 8=\mathrm{R}: \mathrm{C}_{6} \mathrm{H}_{5} \& \mathrm{R}^{1}: \mathrm{C}_{6} \mathrm{H}_{5}$

$D 9=R: H \& R^{1}: H$

$\mathrm{D} 10=\mathrm{R}: \mathrm{C} 6 \mathrm{H}_{5} \& \mathrm{R}^{1}: \mathrm{C}_{6} \mathrm{H}_{5} \mathrm{CHOH}$

\section{RESULTS}

All the Schiff bases synthesized and characterized by above described procedures. Characterization and 
interpretation of all the derived Schiff bases are discussed below.

\section{Solubility}

Solubility of all synthesized Schiff base ligand checked in following solvents like water DMSO, ethanol, acetone and chloroform. The derivatives are frequently soluble in polar solvent and insoluble in non polar solvents.

\section{Characterization of Synthesized Compounds}

\section{D1}

Yield (75\%), m.p. $180-185^{\circ} \mathrm{C}$, Mol. Wt. 1063, Elemental Analysis: (Calculated) for $\mathrm{C}_{58} \mathrm{H}_{54} \mathrm{~N}_{4} \mathrm{O}_{16}$ : ${ }^{\mathrm{C}} \mathrm{C}$, 65.53; H, 5.12; N, 5.27; (Found): C, 65.49; H, 5.18; N, 5.37; FTIR (v, cm-1): $3066(=\mathrm{C}-\mathrm{H}), 1665(\mathrm{C}=\mathrm{N}), 1585$, 1490(C=C, phenyl), 3650(OH), 1692(C=O); ${ }^{1} \mathrm{H}$ NMR

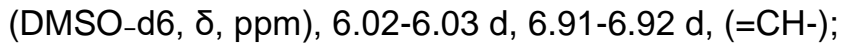
6.88-6.89 t $(=\mathrm{CH}), 2.89-2.90 \mathrm{~d},(-\mathrm{CH}) ; 1.07-1.08 \mathrm{q},(-$ $\left.\mathrm{CH}_{3}\right)$, 3.38-3.39 d; 3.17-3.18 d, $(\mathrm{CH}), 1.47-1.48 \mathrm{t}(\mathrm{CH})$, $1.48 \mathrm{~s},\left(\mathrm{CH}_{3}\right), 6.96-6.97 \mathrm{~d}, 7.577-7.588 \mathrm{~d},(-\mathrm{CH}=)$ $15.21 \mathrm{~s}(\mathrm{OH}) ; 4.62 \mathrm{~s}(\mathrm{OH}) ; 1.46-1.47 \mathrm{~s}(\mathrm{OH}) ;{ }^{13} \mathrm{C}$ NMR (DMSO-d6, $\delta, ~ p p m), ~ 148.4(C 1), ~ 107.8(C 2)$, 132.7(C3), 116.0(C4), 137.3(C5), 40.6(C6), 45.7(C7), 95.6(C8), 174.4(C9), 116.2(C10), 172.2(C11), 68.5(C12), 193.1(C13), 108.5(C14), 195.3(C15), 56.5(C16), 19.0(C17), 68.5(C18), 16.4(C19), 41.8(C20), 41.8(C21), 188.9(C22), 161.6(C23), 136.0(C24), 130.0(C25), 128.2(C26), 130.1(C27), 128.2(C28), 130.0(C29), 161.6(C30), 136.0(C31), 130.0 (C32), 128.2(C33), 130.1(C34), 128.2(C35), 130.0(C36), 188.9(C37), 108.5(C38), 195.3(C39), 56.5(C40), 19.0(C41), 45.7(C42), 68.5(C43), 95.6(C44), 172.2(C45), 68.5(C46), 193.1(C47), 174.4(C48), 116.2(C49), 148.4(C50), 107.8(C51), 132.7(C52), 116.0(C53), 137.3(C54), 40.6(C55), 16.4(C56), 41.8(C57), 41.8(C58).

\section{D2}

Yield (80\%), m.p. $210-213^{\circ} \mathrm{C}$, Mol. Wt. 548; Elemental Analysis: (Calculated) for $\mathrm{C}_{29} \mathrm{H}_{28} \mathrm{~N}_{2} \mathrm{O}_{9}: \mathrm{C}, 63.50 ; \mathrm{H}$, 5.14; N, 5.11; (Found): C, 63.52; H, 5.15; N, 5.20; FTIR $\left(\mathrm{v}, \mathrm{cm}^{-1}\right): 2938(=\mathrm{C}-\mathrm{H}), 1662(\mathrm{C}=\mathrm{N}), 1576(\mathrm{C}=\mathrm{C}$, phenyl), 1771 $(\mathrm{C}=\mathrm{O}), 3649(\mathrm{OH}) ;{ }^{1} \mathrm{H}$ NMR (DMSO-d6, ס, ppm), 6.11-6.12 d, $(=\mathrm{CH}), 6.93 .-6.94 \mathrm{t}(=\mathrm{CH}), 6.90-$ $6.91 \mathrm{~d}, 6.88-6.89 \mathrm{~d}(=\mathrm{CH}), 6.91-6.92,7.05-7.06 \mathrm{t}$ $(=\mathrm{CH})$; 7.51-7.52 d $(=\mathrm{CH})$ 1.07-1.08 q $\left(-\mathrm{CH}_{3}\right)$; 2.872.88d (-CH), 2.49-2.50 t, 3.41-3.42 t (-CH), 2.90-2.91 d $(-\mathrm{CH}-) ; 7.53-7.54 \mathrm{~d}(-\mathrm{C}=\mathrm{N}) ; 1.47 \mathrm{~s}\left(-\mathrm{NCH}_{3}\right) ; 1.459-$ $1.46 \mathrm{~s}(-\mathrm{OH}) ; 4.690-4.7 \mathrm{~s}(\mathrm{OH}) ; 15.202 \mathrm{~s}(\mathrm{OH}) ;{ }^{13} \mathrm{C}$

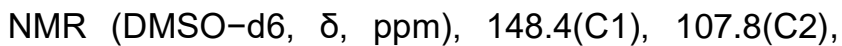
136.9(C3), 119.9(C4), 137.3(C5), 40.6(C6), 41.9(C7), 73.6(C8), 174.4(C9), 117.8(C10), 172.2(C11), 68.4(C12), 192.0 (C13), 95.6(C14), 193 06(C15), 56.48(C16), 19.04(C17), 65.04(C18), 16.34(C19), 40.60(C20), 40.60(C21), 191.0(C22) 161.59(C23), 116.15(C24), 148.4(C25), 115.99(C26), 129.45(C27/29) 122.8(C28), 129.45(C29).

\section{D3}

Yield (85\%), m.p. $190-192^{\circ} \mathrm{C}$; Mol. Wt. 558.58; Elemental Analysis: (Calculated) for $\mathrm{C}_{31} \mathrm{H}_{30} \mathrm{~N}_{2} \mathrm{O}_{8} \mathrm{C}$, 66.66; H, 5.41; N, 5.02; (Found): C, 66.62; H, 5.38; N, 5.01; FTIR (v, $\left.\mathrm{cm}^{-1}\right): \quad 2887(=\mathrm{C}-\mathrm{H}), \quad 1676(\mathrm{C}=\mathrm{N})$, 1549.48(C=C, phenyl) $1709.04(\mathrm{C}=\mathrm{O}), 3468.41(-$ $\mathrm{OH}) ;{ }^{1} \mathrm{H}$ NMR (DMSO-d6, $\left.\delta, \mathrm{ppm}\right), 6.88-6.89 \mathrm{~d}, 6.94-$ $6.95 \mathrm{~d}(=\mathrm{CH}), 6.96-6.97 \mathrm{t}(=\mathrm{CH}) ; 2.81-2.82 \mathrm{~d}(-\mathrm{CH})$, 1.47-1.48 t, 2.61-2.62 t, 3.43-3.42 t (-CH), 2.90-2.92 d $(-\mathrm{CH})$; 4.638-4.639 t $(=\mathrm{CH}-), 1.077-1.078$ q $(-\mathrm{CH} 3)$, $1.46 \mathrm{~s}\left(-\mathrm{N}-\mathrm{CH}_{3}\right) ; 7.42-743 \mathrm{~d}(=\mathrm{CH}), 7.41-7.42 \mathrm{t}(=\mathrm{CH})$, 7.49-7.50 d $(\mathrm{C}=\mathrm{N}) ; 15.10 \mathrm{~s}(-\mathrm{OH}) ; 1.46 \mathrm{~s}(-\mathrm{OH}) ;{ }^{13} \mathrm{C}$ NMR (DMSO-d6, $\delta, \mathrm{ppm}), 153.7(\mathrm{C} 1), 107.8(\mathrm{C} 2)$, 131.7(C3), 116.4(C4), $137.3 \quad$ (C5), 40.6(C6), 45.7(C7), 95.6(C8), 174.2(C9), 116.0(C10), 172.2(C11), 68.5(C12), 193.0(C13), 95.7(C14), 195.0(C15), 56.0(C16), 19.0(C17), 65.0(C18), 19.0(C19), 41.8(C20), 41.8(C21), 194.5(C22), 161.6(C23), 116.16(C24), 129.6(C25), 129.5(C26), 129.0(C27), 128.7(C28), 129.0(C29), 128.7(C30), (128.7C31).

\section{D4}

Yield (77\%), m.p. $210-213^{\circ} \mathrm{C}$, Mol. Wt. 578.57, Elemental Analysis: (Calculated) for $\mathrm{C}_{30} \mathrm{H}_{30} \mathrm{~N}_{2} \mathrm{O}_{10}$ : C, 62.28; H, 5.23; N, 4.84; (Found): C, 62.262; H, 5.22; $\mathrm{N}, 4.83$; FTIR $\left(\mathrm{v}, \mathrm{cm}^{-1}\right): 2366(=\mathrm{CH}-), 1678(\mathrm{C}=\mathrm{N})$, $1577.1 \quad\left(\mathrm{C}=\mathrm{C}\right.$, phenyl), $3299 \quad(\mathrm{OH}) ;{ }^{1} \mathrm{H} \quad \mathrm{NMR}$ (DMSO-d6, $\delta, p p m$ ), 6.03-6.04 d, 6.88-6.89 d $(=\mathrm{CH})$, 6.94-6.95 t $(=\mathrm{CH}), 6.96-6-97 \mathrm{~d}, 7.53-7.54 \mathrm{~d}(=\mathrm{CH})$, $1.46 \mathrm{q}\left(-\mathrm{CH}_{3}\right)$ 1.02-1.03 t, 3.43-3.45 t $(-\mathrm{CH}), 2.89-$ $2.90 \mathrm{~d}, 2.86-2.87 \mathrm{~d}(-\mathrm{CH}) ; 1.48 \mathrm{~s}\left(-\mathrm{CH}_{3}\right)$, 7.537.57 $(=\mathrm{CH}), 6.88-6.89 \mathrm{~d}(\mathrm{C}=\mathrm{C}) .7 .55-7.56 \mathrm{~d}(-\mathrm{CH}=) 15$ $\mathrm{s}(-\mathrm{OH}), 1.47 \mathrm{~s}(-\mathrm{OH}) ;{ }^{13} \mathrm{C}$ NMR (DMSO-d6, $\left.\delta, \mathrm{ppm}\right)$, 148.4(C1), 116.0(C2), 137.22(C3), 116.44(C4), 140.3(C5), 41.1(C6), 45.7(C7), 95.6(C8), 188.0(C9), 116.14(C10), 174.3(C11), 68.50(C12), 193.1(C13), 107.7(C14), 194.2(C15), 56.5(C16), 19.02(C17), 65.1(C18), 16.34(C19), 45.73(C20), 45.73(C21), 190.0(C22), 174.34(C23), 120.0(C24), 116.2(C25), 140.1(C26), 140.1(C27), 116.2(C28), 118.0 (C29), $56.47(\mathrm{C} 30)$. 


\section{D5}

Yield (83\%), m.p. $192-195^{\circ} \mathrm{C}$, Mol. Wt. 484.5, Elemental Analysis: (Calculated) for $\mathrm{C}_{25} \mathrm{H}_{28} \mathrm{~N}_{2} \mathrm{O}_{8}$ : C, 61.97; H, 5.83; N, 5.78; (Found): C, 61.96; H, 5.81; N, 5.76; FTIR $\left(\mathrm{v}, \mathrm{cm}^{-1}\right): 1660(\mathrm{C}=\mathrm{N}), 1553(\mathrm{C}=\mathrm{C}$, phenyl)

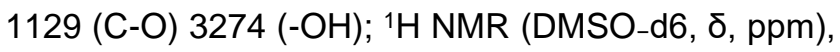
6.885-6.886 d, 6.90-6.91 d, $(=\mathrm{CH})$ 7.53-7.56 t $(=\mathrm{CH})$, 2.901-2.902 d, 3.21-3.22 d (-CH), 1.05-1.06 q $\left(-\mathrm{CH}_{3}\right)$; 3.43-3.45 t, 1.45-1.46 t $(-\mathrm{CH}), 1.46 \mathrm{~s}\left(-\mathrm{N}-\mathrm{CH}_{3}\right), 1.04-$ $1.04 \mathrm{q}\left(-\mathrm{CH}_{3}\right) ; 15.20 \mathrm{~s}(-\mathrm{OH}) ; 1.07 \mathrm{~s}(-\mathrm{OH}) ;{ }^{13} \mathrm{C} \mathrm{NMR}$ (DMSO-d6, $\delta, \quad p p m), \quad 148.4(\mathrm{C} 1), \quad 116.0(\mathrm{C} 2)$, 136.0(C3), 116.4(C4), 137.2(C5), 40.6(C6), 45.7(C7), 95.6(C8), 174.1(C9), 116.20(C10), 172.(C11), 68.5(C12), 187.8(C13), 107.8(C14), 193.1(C15), 56.5(C16), 19.1(C17), 65.1(C18), 16.4(C19), 42.0(C20), 42.0(C21), 190(C22), 161.6(C23), 39.03(C24), 39.03(C25).

\section{D6}

Yield (74\%), m.p. $175-178^{\circ} \mathrm{C}$, Mol. Wt. 546.57, Elemental Analysis: (Calculated) for $\mathrm{C}_{30} \mathrm{H}_{30} \mathrm{~N}_{2} \mathrm{O}_{8}$ : C, 65.92; H, 5.53; N, 5.13; (Found): C, 65.90; H, 5.52; N, 5.14; FTIR (v, cm-1): $2884 \quad(=\mathrm{CH}) \quad 1678(\mathrm{C}=\mathrm{N})$, 1576(C=C, phenyl), 1738(C=O), $3296(-\mathrm{OH}) ;{ }^{1} \mathrm{H}$ NMR

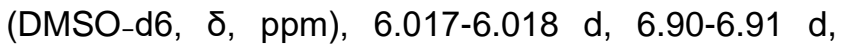
7.57-7.58 d, $(=\mathrm{CH})$ 6.93-6.94 t, 6.95-6-96 t $(=\mathrm{CH}-)$, $1.07 \mathrm{q}\left(-\mathrm{CH}_{3}\right) ; 2.801-2.81 \mathrm{~d},(-\mathrm{CH}), 2.65-2.66 \mathrm{t}, 1.46-$ $1.47 \mathrm{t}, 3.45-3.46 \mathrm{t}(-\mathrm{CH}), 3.41-3.42 \mathrm{~d}(-\mathrm{CH}), 2.53 \mathrm{~s}(-$ $\left.\mathrm{CH}_{3}\right) ; 1.0 \mathrm{~d}(\mathrm{C}=\mathrm{N}), 15 \mathrm{~s}(-\mathrm{OH}), 1.47 \mathrm{~s}(-\mathrm{OH}) ;{ }^{13} \mathrm{C}$ NMR (DMSO-d6, $\delta, p p m), 148.4(\mathrm{C} 1), 107.8(\mathrm{C} 2)$, 133.7(C3), 116.1(C4), 40.6(C6), 45.66(C7), 73.6(C8), 174.3(C9), 116.0(C10), 172.20(C11), 68.4(C12), 194.2(C13), 95.6(C14), 198.4(C15), 56.5(C16), 27.20(C17), 65.1(C18), 16.3(C19), 42.7(C20), 42.7(C21), 193.07(C22), 161.6(C23), 19.0(C24), 129.1(C25), 128.6(C26), 116.4 (C27/29), 131.1(C28), 116.4(C29), 128.6(C30).

\section{D7}

Yield $(79 \%)$, m.p. $170-173^{\circ} \mathrm{C}$, Mol. Wt. 532.54, Elemental Analysis: (Calculated) for $\mathrm{C}_{29} \mathrm{H}_{28} \mathrm{~N}_{2} \mathrm{O}_{8}$ : C, 65.41; H, 5.30; N, 5.26; (Found): C, 65.40; H, 5.28; N, 5.25; FTIR (v, cm$\left.{ }^{-1}\right): 2820(=\mathrm{CH}), \quad 1660(\mathrm{C}=\mathrm{N})$, 1612(C=C) $1578\left(\mathrm{C}=\mathrm{C}\right.$, phenyl) $3267(-\mathrm{OH}) ;{ }^{1} \mathrm{H}$ NMR (DMSO-d6, $\delta, ~ p p m), 6.19-6.20 \mathrm{~d}, 688-6.89 \mathrm{~d}, 7.56-$ $7.57 \mathrm{~d}(=\mathrm{CH}-), 6.90-6.91 \mathrm{t} ; 6.95-6.96 \mathrm{t}(=\mathrm{CH}-), 1.07 \mathrm{q}$ $\left(-\mathrm{CH}_{3}\right), 2.90-2.91 \mathrm{~d}, 3.16-3.17 \mathrm{~d},(-\mathrm{CH}) ; 2.66-2.67 \mathrm{t}$; 2.64-2.65 t; 3.16-3.17 t $(-\mathrm{CH}), 1.45 \mathrm{~s}\left(-\mathrm{CH}_{3}\right)$; 7.95$7.96 \mathrm{~d}(=\mathrm{CH}) 15.8 \mathrm{~s}(-\mathrm{OH}) 4.67 \mathrm{~s}(\mathrm{OH}), 1.458 \mathrm{~s}(-$ $\mathrm{OH}) ;{ }^{13} \mathrm{C}$ NMR (DMSO-d6, $\left.\delta, \mathrm{ppm}\right), 148.37(\mathrm{C} 1)$,
107.8(C2), 133.3(C3), 116.4(C4), 137.2(C5), 41.9(C6), 45.7(C7), 73.6(C8), 174.1(C9), 116.1(C10), 172.2(C11), 68.4(C12), 193.0(C13), 95.58(C14), 194.2(C15), 56.4(C16), 19.0(C17), 65.0(C18), 16.3(C19), 41.94(C20/21), 41.94(C21), 188.0(C22), 161.6(C23), 129.9(C24), 129.72(C25), 129.0(C26), 129.6(C27), 129.0(C28), 129.72(C29).

\section{D8}

Yield (83\%), m.p. $179-181^{\circ} \mathrm{C}$, Mol. Wt. 608.64, Elemental Analysis: (Calculated) for $\mathrm{C}_{35} \mathrm{H}_{32} \mathrm{~N}_{2} \mathrm{O}_{8}$ : C, 69.07; H, 5.30; N, 4.60; (Found): C, 69.07; H, 5.30; N, 4.59; FTIR (v, cm $\left.{ }^{-1}\right): 3058(=\mathrm{CH})$ 1657(C=N), 1576 $\left(\mathrm{C}=\mathrm{C}\right.$, phenyl) $1612(\mathrm{C}=\mathrm{C}) 3275(-\mathrm{OH}) ;{ }^{1} \mathrm{H}$ NMR (DMSO-d6, $\delta$, ppm), 6.05-6.06 d; 6.88-6.89 d, 7.71$7.72 \mathrm{~d} ; 7.73-7.74 \mathrm{~d}(=\mathrm{CH}), 6.904 \mathrm{t}, 6.91 \mathrm{t}, 6.94-6-95 \mathrm{t}$; 6.95-6.96 t $(=\mathrm{CH}-), 2.87-2.88 \mathrm{~d}$; 3.17-3.18 d $(-\mathrm{CH})$, 3.45-3.46 t; $1.45-1.46 \mathrm{t} ; 1.47-1.48 \mathrm{t}(-\mathrm{CH}), 1.03 \mathrm{q}(-$ $\left.\mathrm{CH}_{3}\right) ; 1.07-1.08 \mathrm{~s}\left(-\mathrm{CH}_{3}\right), 15 \mathrm{~s}(-\mathrm{OH}) ; 1.03 \mathrm{~s}(-\mathrm{OH})$, $4.7 \mathrm{~s}(\mathrm{OH}) ;{ }^{13} \mathrm{C}$ NMR (DMSO-d6, $\left.\delta, \mathrm{ppm}\right), 148.4(\mathrm{C} 1)$, 107.8(C2), 137.2(C3), 116.0(C4), 137.5(C5), 40.6(C6), 45.7(C7), 73.6(C8), 174.4(C9), 116.1(C10), 172.2(C11), 68.4(C12), 193.1(C13), 96.6(C14), 196.3(C15), 56.5(C16), 19.0(C17), 65.1(C18), 16.3(C19), 41.83(C20), 41.83(C21), 190.0(C22), 161.5(C23), 130.1(C24), 129.0(C25), 116.4(C26), 133.2(C27), 129.0(C29), 130.1(C30), 129.0(C31), 116.4(C32), 133.2(С33), 129.0(C34), 129.0(С35).

\section{D9}

Yield (81\%), m.p. $185-192^{\circ} \mathrm{C}$, Mol. Wt. 456.45, Elemental Analysis: (Calculated) for $\mathrm{C}_{23} \mathrm{H}_{24} \mathrm{~N}_{2} \mathrm{O}_{8}$ : C, 60.52; H, 5.30; N, 6.14; (Found): C, 60.50; H, 5.31; N, 6.13; FTIR (v, cm$\left.{ }^{-1}\right): 3058(=\mathrm{CH}), 1657(\mathrm{C}=\mathrm{N}), 1576$ $(\mathrm{C}=\mathrm{C}$, phenyl) 1612, $(\mathrm{C}=\mathrm{C}) 1740(\mathrm{C}=\mathrm{O}) 3275(-\mathrm{OH})$; ${ }^{1} \mathrm{H}$ NMR (DMSO-d6, $\delta, \mathrm{ppm}$ ), 6.17-6.18 d; 6..88-.6.89 $\mathrm{d}(=\mathrm{CH}), 6.90-6.91 \mathrm{t}(-\mathrm{CH}), 2.90-2.91 \mathrm{~d}, 3.168-3.17 \mathrm{~d}$ $(-\mathrm{CH}), 1.47-1.48 \mathrm{t} ; 1.45-1.46 \mathrm{t}, 3.41-3.42 \mathrm{t}(-\mathrm{CH})$, 1.03-1.04 q $(-\mathrm{CH} 3), 1.07-1.08 \mathrm{~s}\left(-\mathrm{CH}_{3}\right), 15.20 \mathrm{~s}$ $(-\mathrm{OH}), \quad 1.0 \mathrm{~s} \quad(-\mathrm{OH}), \quad 4.72 \mathrm{~s}(\mathrm{OH}) ;{ }^{13} \mathrm{C} \quad \mathrm{NMR}$ (DMSO-d6, $\delta, \quad p p m), \quad 148.4(\mathrm{C} 1), \quad 107.8(\mathrm{C} 2)$, 116.4(C3), 116.2(C4), 137.23(C5), 40.9(C6), 45.7(C7), $\quad 73.60(\mathrm{C} 8), \quad 174.4(\mathrm{C} 9), \quad 116.00(\mathrm{C} 10)$, 172.2(C11), 68.4(C12), 188.0(C13), 95.6(C14), 193.1(C15), 56.5(C16), 19.0(C17), 65.0(C18), 16.4(C19), 41.92(C20), 41.92(C21), 190.0(C22), 161.6(C23). 
D10

Yield (72\%), m.p. $185-187^{\circ} \mathrm{C}$, Mol. Wt. 638.66, Elemental Analysis: (Calculated) for $\mathrm{C}_{36} \mathrm{H}_{34} \mathrm{~N}_{2} \mathrm{O}_{9}$ : C, 67.70; H, 5.37; N, 4.39; (Found): C, 67.71; H, 5.35; N, 4.3; 7FTIR (, $\left.\mathrm{cm}^{-1}\right): 2972(=\mathrm{C}-\mathrm{H}), 1611.11(\mathrm{C}=\mathrm{C})$, 1660 $(\mathrm{C}=\mathrm{N}), 1577.96$ (C=C, phenyl) 3224(-OH); ${ }^{1} \mathrm{H}$ NMR (DMSO-d6, $\delta, \mathrm{ppm}$ ), 5.99-6.00d; 6.88.-6.89 d, 6.95-6.96 d 7.572-7.58 d $(=\mathrm{CH}), 6.92-6.93$ t 6.90-6.91 $\mathrm{t} ; 6.94-6.95 \mathrm{t}(-\mathrm{CH}), 2.71-2.72 \mathrm{t}, 1.47-1.48 \mathrm{t} ; 3.41-3.42$ t 2.82-2.83 d, 3.35-3.36 d (-CH), 1,07-1.08 q $\left(-\mathrm{CH}_{3}\right)$, $1.46 \mathrm{~s}\left(\mathrm{CH}_{3}\right), 1.052(\mathrm{OH}), 4.5 \mathrm{~s}(\mathrm{OH}), 15.50 \mathrm{~s}(\mathrm{OH})$; ${ }^{13} \mathrm{C}$ NMR (DMSO-d6, $\left.\delta, \mathrm{ppm}\right), 161.6(\mathrm{C} 1), 113.6(\mathrm{C} 2)$, 135.8(C3), 121.3(C4), 141.2(C5), 40.2(C6), 45.7(C7), 106.6(C8), 183.1(C9), 119.6(C10), 174.9(C11), 68.2(C12), 193.1(C13), 110.7(C14), 202.1(C15),
63.5(C16), 32.4(C17), 67.9(C18), 19.4(C19), 44.4(C20), 44.4(C21), 190(C22), 164.6(C23), 152.5(C24), 141.6(C25), 127.2(C26), 129.2(C27), 127.2(C28), 129.2(C29), 129.2(С30), 134.0(C31), 129.2(С32), 128.9(С33), 131.1(С34), 128.9(С35), 129.2(C36).

\section{Antibacterial Activity}

Antibacterial action of all the prepared Schiff base ligands were checked by agar well diffusion method against the following strains (Table 2a, 2b); I, Bacillus subtilis II, Micrococcus luteus III, Staphylococcus aureus IV, Stenotrophomonas maltophilia V, Serratia marcescens VI and Escherichia coli VII.

Table 2a. Zone of inhibition of Schiff bases against bacterial strains.

\begin{tabular}{|c|c|c|c|c|c|c|c|c|}
\hline \multirow{4}{*}{$\begin{array}{c}\text { Derivatives } \\
\text { D1 }\end{array}$} & \multicolumn{2}{|c|}{$\begin{array}{c}\text { Bacillus } \\
\text { megaterium I }\end{array}$} & \multicolumn{2}{|c|}{ Bacillus subtilis II } & \multicolumn{2}{|c|}{ Micrococcus luteus III } & \multicolumn{2}{|c|}{$\begin{array}{c}\text { Staphylococcus } \\
\text { aureus IV }\end{array}$} \\
\hline & A & $24 \pm 2.0$ & A & $32 \pm 1.5$ & A & $34 \pm 0.9$ & A & $31 \pm 0.5$ \\
\hline & $\mathrm{B}$ & $13 \pm 0.5$ & $\mathrm{~B}$ & $26 \pm 1$ & $\mathrm{~B}$ & $26 \pm 0.3$ & $\mathrm{~B}$ & $26 \pm 1.0$ \\
\hline & $\mathrm{C}$ & $10 \pm 0.0$ & $\mathrm{C}$ & $17 \pm 1$ & $\mathrm{C}$ & $13 \pm 0.4$ & $\mathrm{C}$ & $17 \pm 1.0$ \\
\hline \multirow{3}{*}{$\mathrm{D} 2$} & A & $18 \pm 0.5$ & A & $39 \pm 3.5$ & A & $35 \pm 0.7$ & A & $33 \pm 0.575$ \\
\hline & B & $11 \pm 1.0$ & $B$ & $30 \pm 3.4$ & $\mathrm{~B}$ & $28 \pm 0.2$ & $\mathrm{~B}$ & $23 \pm 05$ \\
\hline & $\mathrm{C}$ & $8 \pm 1.5$ & $\mathrm{C}$ & $19 \pm 2.0$ & $\mathrm{C}$ & $15 \pm 0$ & $\mathrm{C}$ & $12 \pm 2.0$ \\
\hline \multirow{3}{*}{ D3 } & A & $18 \pm 0.5$ & A & $28 \pm 1.2$ & $\mathrm{~A}$ & $19 \pm 0.8$ & A & $25 \pm 1.0$ \\
\hline & $\mathrm{B}$ & $11 \pm 1.0$ & $\mathrm{~B}$ & $19 \pm 1.82$ & $\mathrm{~B}$ & $10 \pm 0.0$ & $\mathrm{~B}$ & $16 \pm 0.6$ \\
\hline & $\mathrm{C}$ & $8 \pm 1.0$ & $\mathrm{C}$ & $10 \pm 1.8$ & $\mathrm{C}$ & $5 \pm 0.0$ & $\mathrm{C}$ & $8 \pm 0.7$ \\
\hline \multirow{3}{*}{ D4 } & A & $20 \pm 1.0$ & A & $33 \pm 2.8$ & $\mathrm{~A}$ & $25 \pm 0.5$ & $A$ & $30 \pm 1.8$ \\
\hline & $B$ & $17 \pm 1.0$ & $B$ & $26 \pm 1.9$ & $\mathrm{~B}$ & $20 \pm 0.9$ & $B$ & $20 \pm 2.02$ \\
\hline & $\mathrm{C}$ & $10 \pm .56$ & $\mathrm{C}$ & $15 \pm 1.0$ & $C$ & $14 \pm 2.0$ & $\mathrm{C}$ & $10 \pm 1.0$ \\
\hline \multirow{3}{*}{ D5 } & A & $22 \pm 78$ & A & $30 \pm 2.4$ & A & $29 \pm 0.9$ & A & $31 \pm 2.05$ \\
\hline & $B$ & $16 \pm 0.67$ & B & $19 \pm 1.9$ & $B$ & $21 \pm 0.4$ & $\mathrm{~B}$ & $27 \pm 2.8$ \\
\hline & $C$ & $9 \pm 0.8$ & $\mathrm{C}$ & $10 \pm 1.2$ & $\mathrm{C}$ & $10 \pm 0.0$ & $C$ & $14 \pm 1.5$ \\
\hline \multirow{3}{*}{ D6 } & $A$ & $21 \pm 1.5$ & $A$ & $30 \pm 1.09$ & $A$ & $30 \pm 0.1$ & $A$ & $38 \pm 0.5$ \\
\hline & $B$ & $16 \pm 0.7$ & B & $21 \pm 2.0$ & $\mathrm{~B}$ & $22 \pm 0.5$ & $\mathrm{~B}$ & $30 \pm 1.5$ \\
\hline & $C$ & $10 \pm .57$ & $\mathrm{C}$ & $11 \pm 1.4$ & $\mathrm{C}$ & $11 \pm 0.2$ & $C$ & $16 \pm .75$ \\
\hline \multirow{3}{*}{ D7 } & $A$ & $22 \pm 1.2$ & $A$ & $27 \pm 1.5$ & $A$ & $36 \pm 2$ & $A$ & $39 \pm 0.5$ \\
\hline & $B$ & $19 \pm 1.6$ & $B$ & $23 \pm 0.5$ & $B$ & $24 \pm 1$ & $B$ & $26 \pm 0.5$ \\
\hline & C & $9 \pm 0.8$ & C & $12 \pm 2.0$ & C & $12 \pm 0.5$ & C & $15 \pm 0.5$ \\
\hline \multirow{3}{*}{ D8 } & $A$ & $22 \pm .75$ & A & $32 \pm 0.8$ & $A$ & $35 \pm 2$ & $A$ & $25 \pm 0.5$ \\
\hline & $B$ & $19 \pm 1.5$ & $B$ & $26 \pm 1.2$ & $\mathrm{~B}$ & $29 \pm 0.5$ & $B$ & $16 \pm 0.5$ \\
\hline & $C$ & $11 \pm 1.0$ & $\mathrm{C}$ & $14 \pm 2.0$ & $\mathrm{C}$ & $15 \pm 1$ & $\mathrm{C}$ & $8 \pm 0.5$ \\
\hline \multirow{3}{*}{ D9 } & A & $23 \pm .75$ & A & $28 \pm 2.0$ & A & $28 \pm 0.6$ & A & $29 \pm 0.5$ \\
\hline & $B$ & $17 \pm 1.0$ & $B$ & $24 \pm 1.9$ & $B$ & $18 \pm 0.5$ & $B$ & $21 \pm 0.5$ \\
\hline & C & $9 \pm 0.75$ & C & $13 \pm 1.0$ & $\mathrm{C}$ & $9 \pm 0.3$ & $\mathrm{C}$ & $11 \pm 0.5$ \\
\hline \multirow{3}{*}{ D10 } & A & $23 \pm 0.9$ & $A$ & $33 \pm 2.09$ & $A$ & $20 \pm 1$ & $A$ & $28 \pm 0.5$ \\
\hline & $B$ & $16 \pm 0.5$ & $B$ & $26 \pm 2.0$ & $B$ & $12 \pm 0.5$ & $B$ & $19 \pm 0.5$ \\
\hline & $C$ & $10 \pm 1.8$ & $\mathrm{C}$ & $14 \pm 1.0$ & $\mathrm{C}$ & $7 \pm 0.6$ & C & $10 \pm 0.5$ \\
\hline
\end{tabular}


Table $2 \mathrm{~b}$. Zone of inhibition of Schiff bases against bacterial strains.

\begin{tabular}{|c|c|c|c|c|c|c|}
\hline \multirow{4}{*}{$\begin{array}{c}\text { Derivatives } \\
\text { D1 }\end{array}$} & \multicolumn{2}{|c|}{$\begin{array}{c}\text { Stenotrophomonas } \\
\text { maltophilia V }\end{array}$} & \multicolumn{2}{|c|}{ Serratia marcescens VI } & \multicolumn{2}{|c|}{ Escherichia coli VII } \\
\hline & A & $24 \pm 0.9$ & $A$ & $25 \pm 0.7$ & A & $37 \pm 0.9$ \\
\hline & $\mathrm{B}$ & $15 \pm 0.3$ & $\mathrm{~B}$ & $16 \pm 0.8$ & $\mathrm{~B}$ & $28 \pm 0.5$ \\
\hline & $\mathrm{C}$ & $8 \pm 0.4$ & $\mathrm{C}$ & $8 \pm 0.9$ & C & $18 \pm 0.7$ \\
\hline \multirow{3}{*}{ D2 } & $\mathrm{A}$ & $31 \pm 0.7$ & A & $27 \pm 0.3$ & $\mathrm{~A}$ & $28 \pm 0.00$ \\
\hline & $\mathrm{B}$ & $20 \pm 0.2$ & $\mathrm{~B}$ & $20 \pm 0.9$ & $\mathrm{~B}$ & $22 \pm 1.0$ \\
\hline & C & $10 \pm 0$ & $\mathrm{C}$ & $9 \pm 0.9$ & C & $11 \pm 0.5$ \\
\hline \multirow{3}{*}{ D3 } & A & $41 \pm 0.8$ & A & $19 \pm 2.0$ & A & $29 \pm 0.4$ \\
\hline & $\mathrm{B}$ & $34 \pm 0.0$ & $\mathrm{~B}$ & $11 \pm 3.0$ & $\mathrm{~B}$ & $23 \pm 0.3$ \\
\hline & $\mathrm{C}$ & $18 \pm 0.0$ & $\mathrm{C}$ & $5 \pm 3.0$ & $\mathrm{C}$ & $16 \pm 0.5$ \\
\hline \multirow{3}{*}{ D4 } & $A$ & $37 \pm 0.5$ & $A$ & $23 \pm 0.8$ & A & $29 \pm 0.7$ \\
\hline & $\mathrm{B}$ & $29 \pm 0.9$ & $\mathrm{~B}$ & $17 \pm 0.7$ & $\mathrm{~B}$ & $20 \pm 0.5$ \\
\hline & C & $15 \pm 2.0$ & $\mathrm{C}$ & $9 \pm 0.9$ & $\mathrm{C}$ & $10 \pm 0.6$ \\
\hline \multirow{3}{*}{ D5 } & A & $38 \pm 0.9$ & A & $28 \pm 0.5$ & A & $27 \pm 0.9$ \\
\hline & B & $30 \pm 0.4$ & $\mathrm{~B}$ & $19 \pm 0.11$ & $B$ & $21 \pm 0.8$ \\
\hline & C & $16 \pm 0.0$ & C & $10 \pm 0.8$ & C & $11 \pm 0.5$ \\
\hline \multirow{3}{*}{ D6 } & $\mathrm{A}$ & $33 \pm 0.1$ & $\mathrm{~A}$ & $25 \pm 0.5$ & $\mathrm{~A}$ & $30 \pm 0.5$ \\
\hline & $B$ & $27 \pm 0.5$ & $\mathrm{~B}$ & $16 \pm 0.5$ & $\mathrm{~B}$ & $21 \pm 0.7$ \\
\hline & C & $14 \pm 0.2$ & $\mathrm{C}$ & $8 \pm 0.7$ & C & $10 \pm 0.7$ \\
\hline \multirow{3}{*}{ D7 } & $\mathrm{A}$ & $30 \pm 2$ & $\mathrm{~A}$ & $29 \pm 0.5$ & $\mathrm{~A}$ & $36 \pm 0.0$ \\
\hline & $B$ & $26 \pm 1$ & $\mathrm{~B}$ & $20 \pm 0.5$ & $B$ & $29 \pm 2$ \\
\hline & C & $14 \pm 0.5$ & C & $9 \pm 0.4$ & C & $17 \pm 1$ \\
\hline \multirow{3}{*}{ D8 } & A & $30 \pm 2$ & A & $33 \pm 0.1$ & A & $34 \pm 2$ \\
\hline & $B$ & $25 \pm 0.5$ & $\mathrm{~B}$ & $26 \pm 0.1$ & $B$ & $26 \pm 1$ \\
\hline & $\mathrm{C}$ & $15 \pm 1$ & C & $17 \pm 0$ & $\mathrm{C}$ & $13 \pm 0.7$ \\
\hline \multirow{3}{*}{ D9 } & $\mathrm{A}$ & $27 \pm 0.6$ & $\mathrm{~A}$ & $30 \pm 0.8$ & A & $36 \pm 0.3$ \\
\hline & $\mathrm{B}$ & $20 \pm 0.5$ & $\mathrm{~B}$ & $24 \pm 0.5$ & $\mathrm{~B}$ & $24 \pm 01$ \\
\hline & $\mathrm{C}$ & $11 \pm 0.3$ & C & $12 \pm 0.0$ & $\mathrm{C}$ & $17 \pm 0$ \\
\hline \multirow{3}{*}{ D10 } & A & $28 \pm 1$ & A & $27 \pm 0.7$ & A & $28 \pm 1$ \\
\hline & B & $15 \pm 0.5$ & B & $18 \pm 0.4$ & B & $20 \pm 0.6$ \\
\hline & C & $10 \pm 0.6$ & C & $12 \pm 0.5$ & $\mathrm{C}$ & $10 \pm 0.4$ \\
\hline
\end{tabular}

Alphabets $A B C$ indicates concentration of novel Schiff bases $5 \mathrm{mg} / \mathrm{ml}, 2.5 \mathrm{mg} / \mathrm{ml}$ and $1 \mathrm{mg} / \mathrm{ml}$ respectively against sevens trains.

Table 3. Standard drug against bacterial strains.

\begin{tabular}{|c|c|c|c|c|c|c|c|}
\hline \multicolumn{7}{|c|}{ Inhibitory Area of Standard Drug in millimeters } \\
\hline Drug & $\begin{array}{c}\text { Bacillus } \\
\text { megaterium } \\
\text { I }\end{array}$ & $\begin{array}{c}\text { Bacillus } \\
\text { subtilis } \\
\text { II }\end{array}$ & $\begin{array}{c}\text { Micrococcus } \\
\text { Iuteus III }\end{array}$ & $\begin{array}{c}\text { Staphylococcus } \\
\text { aureus IV }\end{array}$ & $\begin{array}{c}\text { Stenotrophomonas } \\
\text { maltophilia V }\end{array}$ & $\begin{array}{c}\text { Serratia } \\
\text { marcescens } \\
\text { VI }\end{array}$ & $\begin{array}{c}\text { Escherichia } \\
\text { coli VII }\end{array}$ \\
\hline A & $25 \pm 0.3$ & $32 \pm 0.1$ & $28 \pm 0.00$ & $30 \pm 0.2$ & $28 \pm 0.2$ & $20 \pm 0.01$ & $35 \pm 0.1$ \\
\hline B & $15 \pm 0.4$ & $20 \pm 0.3$ & $18 \pm 0.1$ & $25 \pm 0.3$ & $21 \pm 0.3$ & $15 \pm 0.02$ & $24 \pm 0.1$ \\
\hline C & $10 \pm 0.2$ & $13 \pm 0.5$ & $12 \pm 0.05$ & $16 \pm 0.4$ & $16 \pm 0.1$ & $8 \pm 0.05$ & $18 \pm 0.3$ \\
\hline
\end{tabular}

Antimicrobial activity of standard drug doxycycline against seven strains are mentioned in Table 3. 


\section{Zone of inhibition against Bacillus megaterium (Graph 1).}

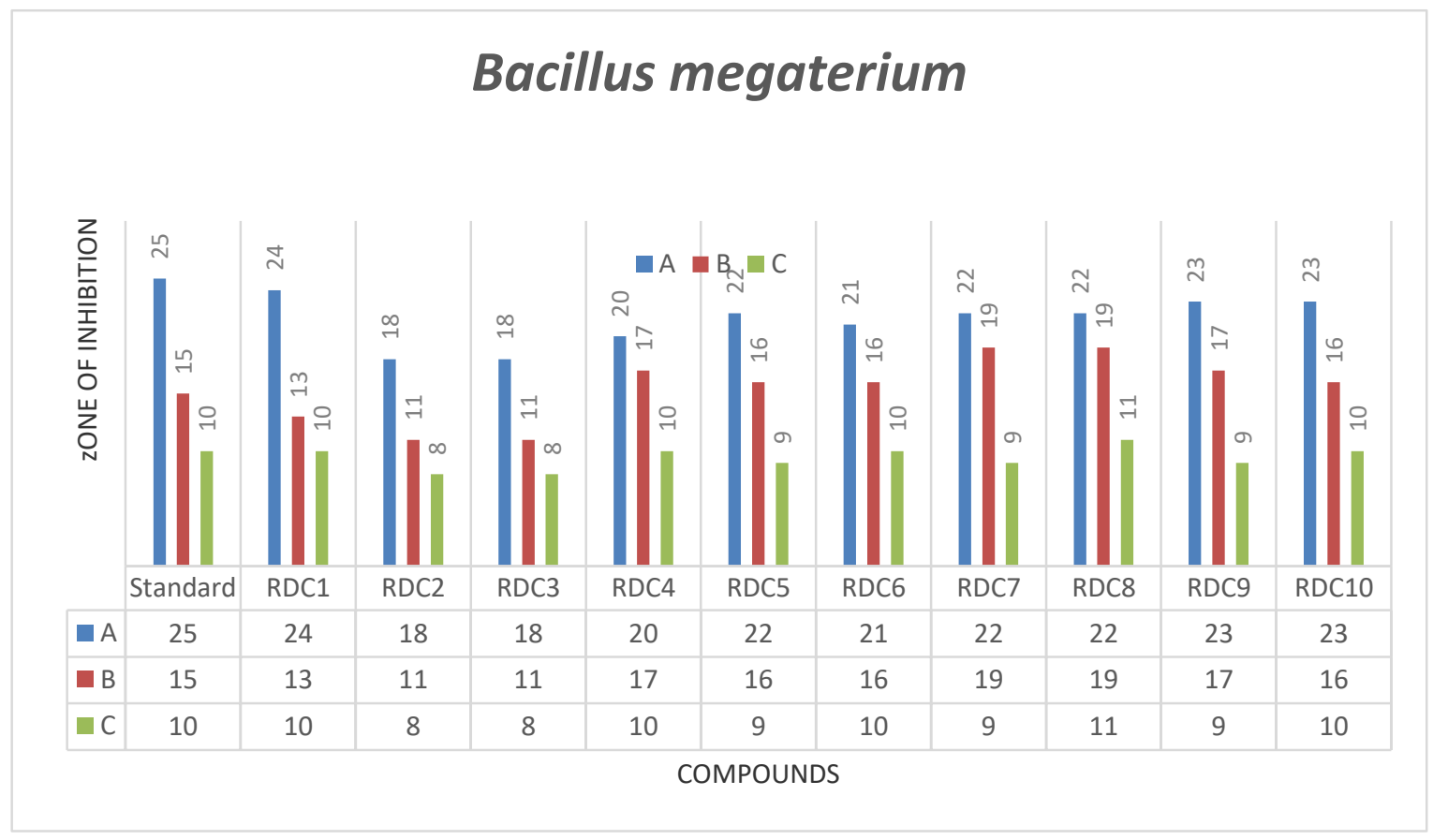

Graph 1. Comparative study of derived Schiff bases vs. doxycycline against Bacillus megaterium Derived Schiff bases showed less zone of inhibition against Bacillus megaterium as compared to doxycycline.

\section{Zone of inhibition against Bacillus subtilis (Graph 2).}

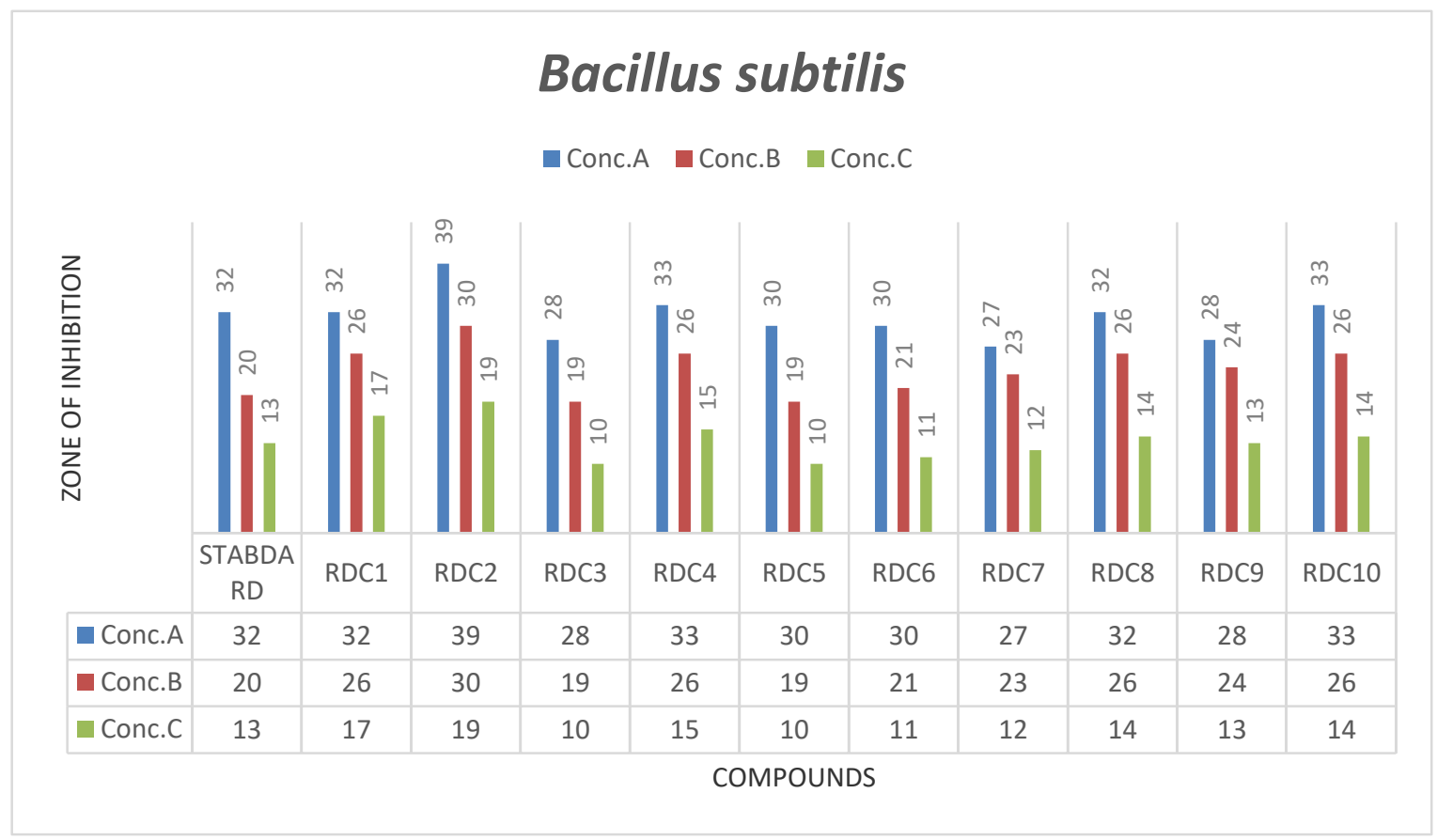

Graph 2. Comparative study of derived Schiff bases vs. doxycycline against Bacillus subtilis.

Derived Schiff bases RDC2, RDC4 and RDC10 showed zone of inhibition against Bacillus subtilis as compared to doxycycline. 


\section{Zone of inhibition against Micrococcus luteus (Graph 3).}

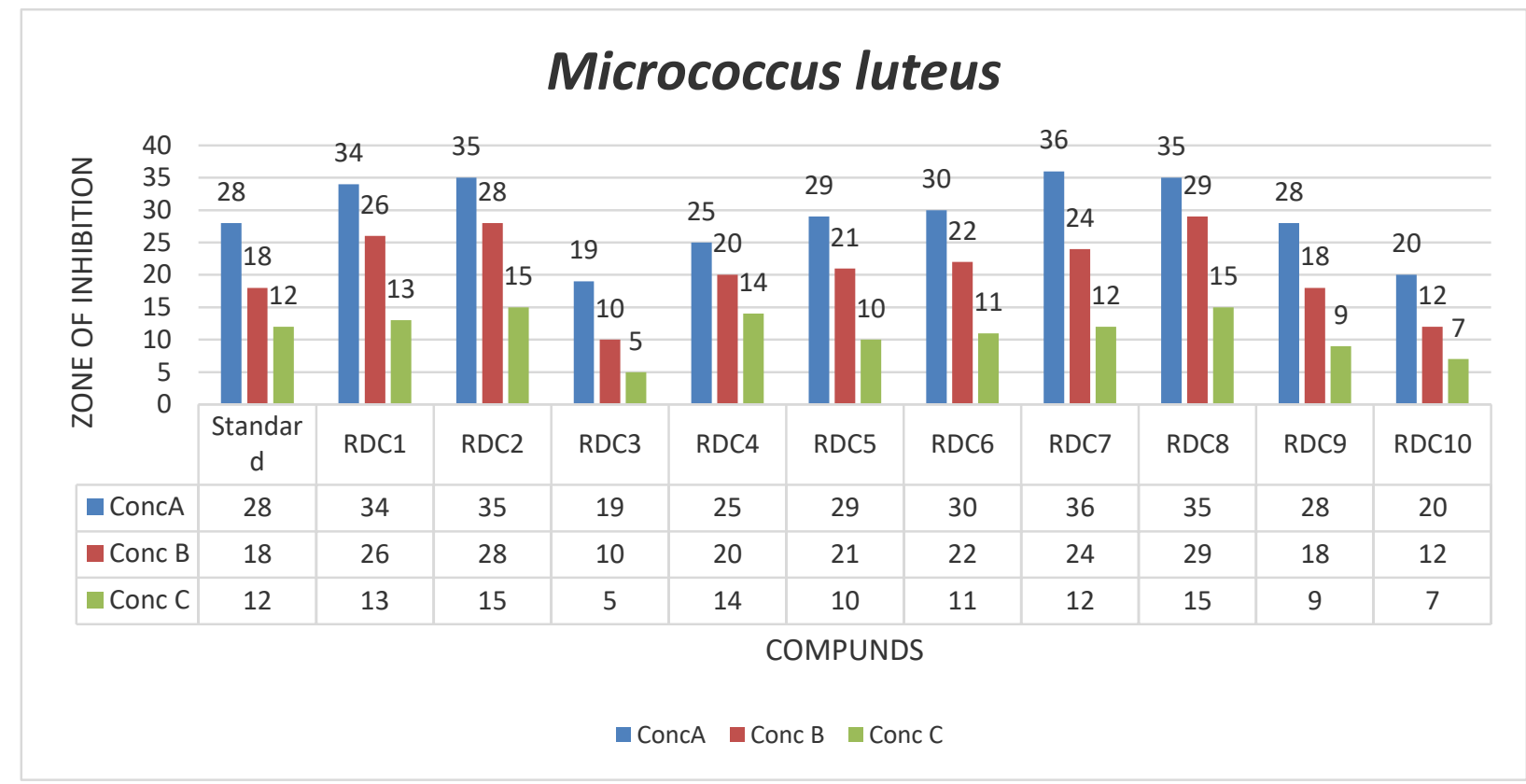

Graph 3. Comparative study of derived Schiff bases vs. doxycycline against Micrococcus luteus.

Derived Schiff bases RDC1, RDC2, RDC5, RDC6, RDC7 and RDC8 showed zone of inhibition against Micrococcus luteus as compared to doxycycline.

\section{Zone of inhibition against Staphylococcus aureus (Graph 4).}

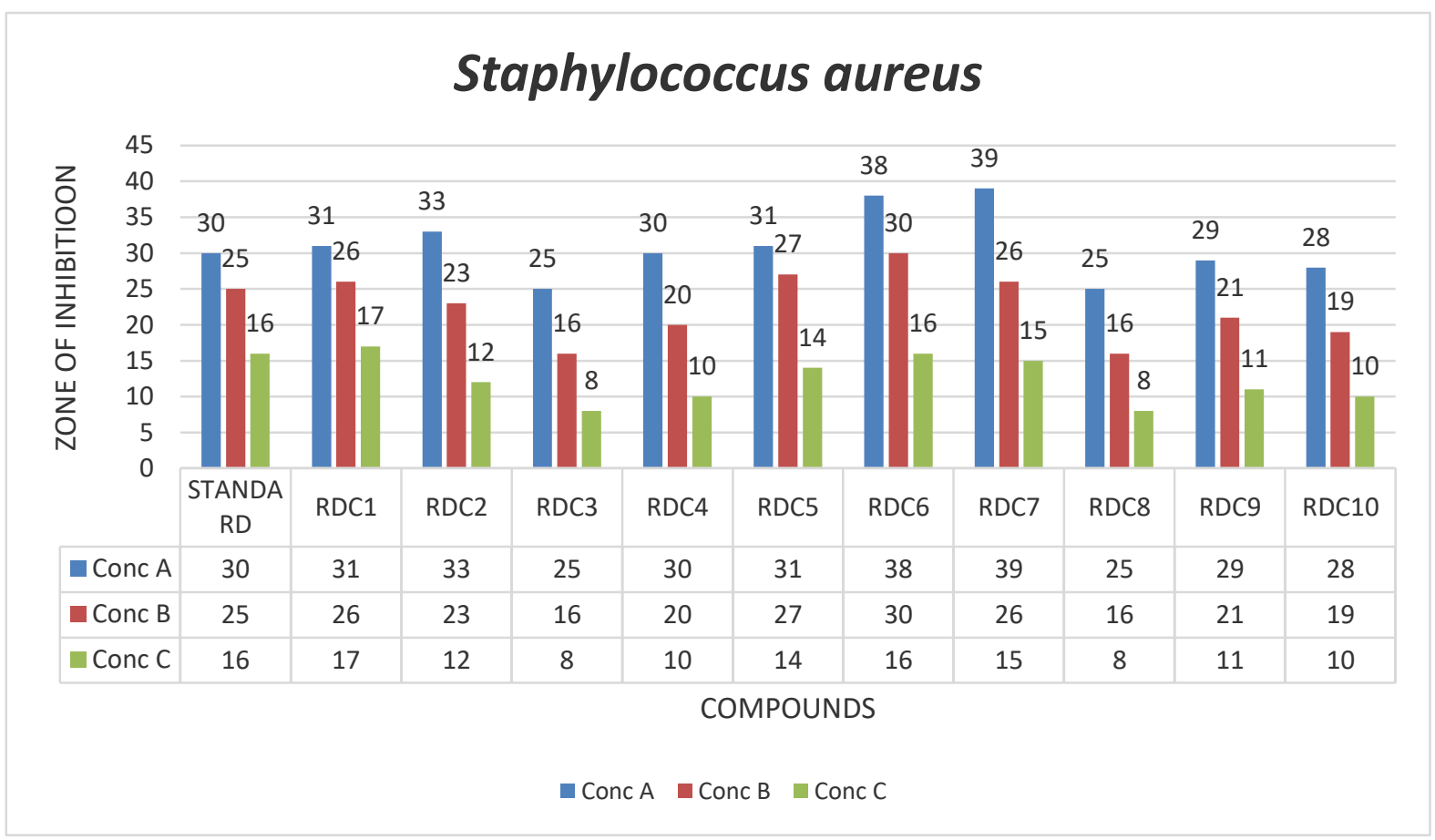

Graph 4. Comparative study of derived Schiff bases vs. doxycycline against Staphylococcus aureus.

Derived Schiff bases RDC1, RDC2, RDC5, RDC6, and RDC7 showed zone of inhibition against Staphylococcus aureus as compared to doxycycline. 


\section{Zone of inhibition against Stenotrophomonas maltophilia (Graph 5).}

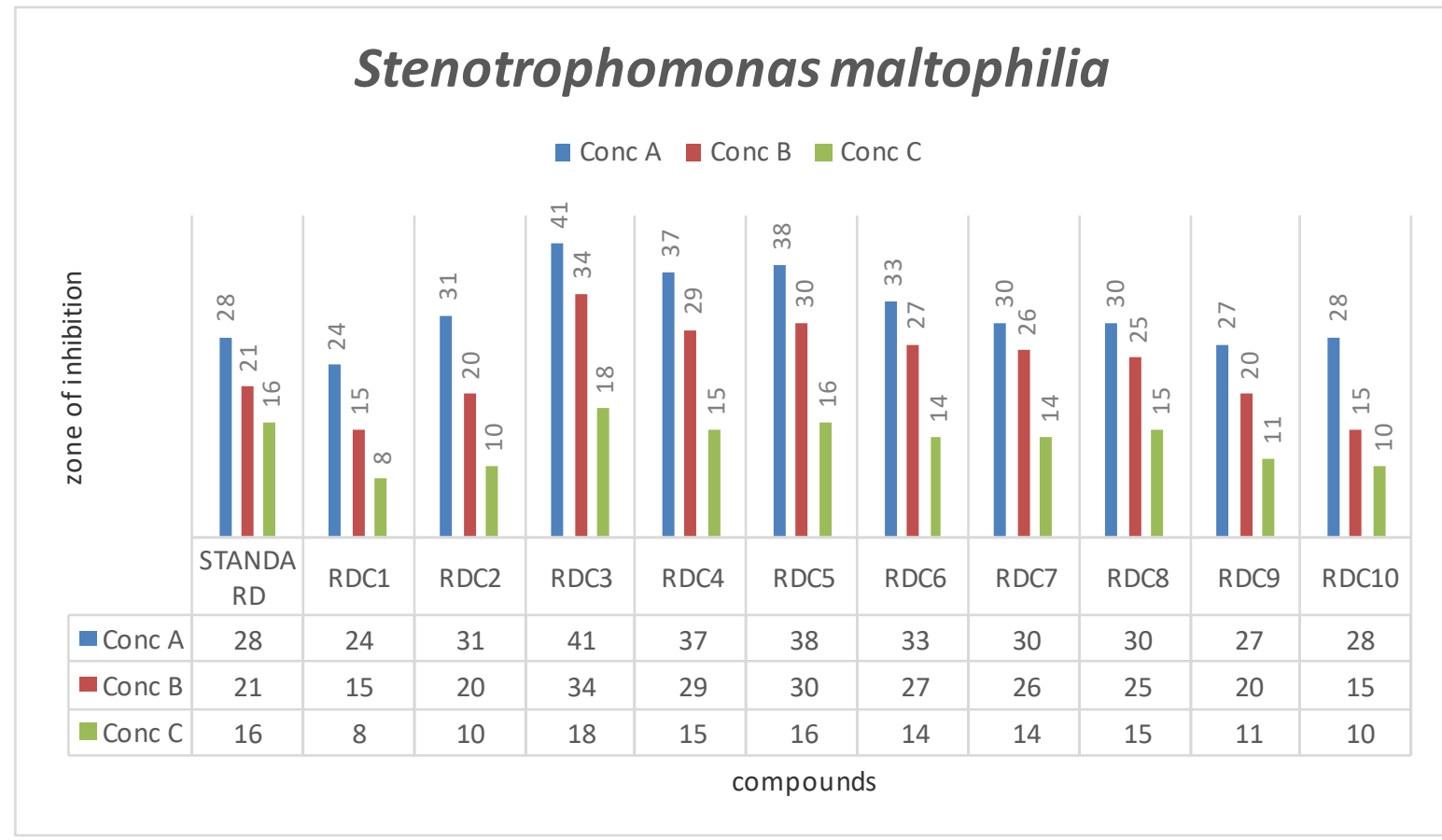

Graph 5. Comparative study of derived Schiff bases vs. doxycycline against Stenotrophomonas maltophilia.

Derived Schiff bases RDC2, RDC3, RDC4, RDC5, RDC6, RDC7 and RDC8 showed zone of inhibition against Stenotrophomonas maltophilia as compared to doxycycline.

\section{Zone of inhibition against Serratia marcescens (Graph 6).}

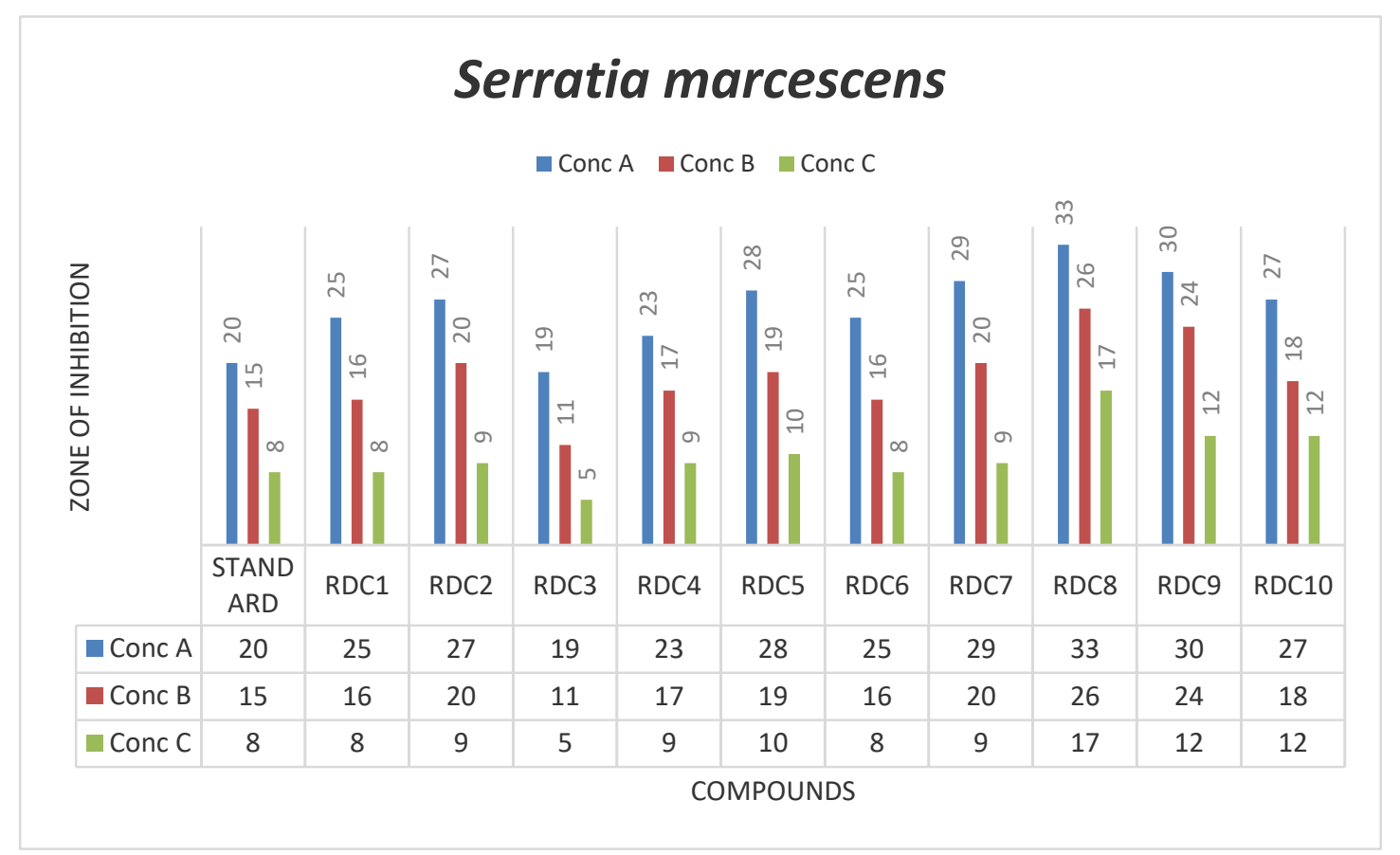

Graph 6. Comparative study of derived Schiff bases vs. doxycycline against Serratia marcescens.

Derived Schiff bases RDC1, RDC2, RDC3, RDC5, RDC6, RDC7, RDC8, RDC9 and RDC10 showed zone of inhibition against Serratia marcescens as compared to doxycycline. 


\section{Zone of inhibition against Escherichia coli (Graph 7).}

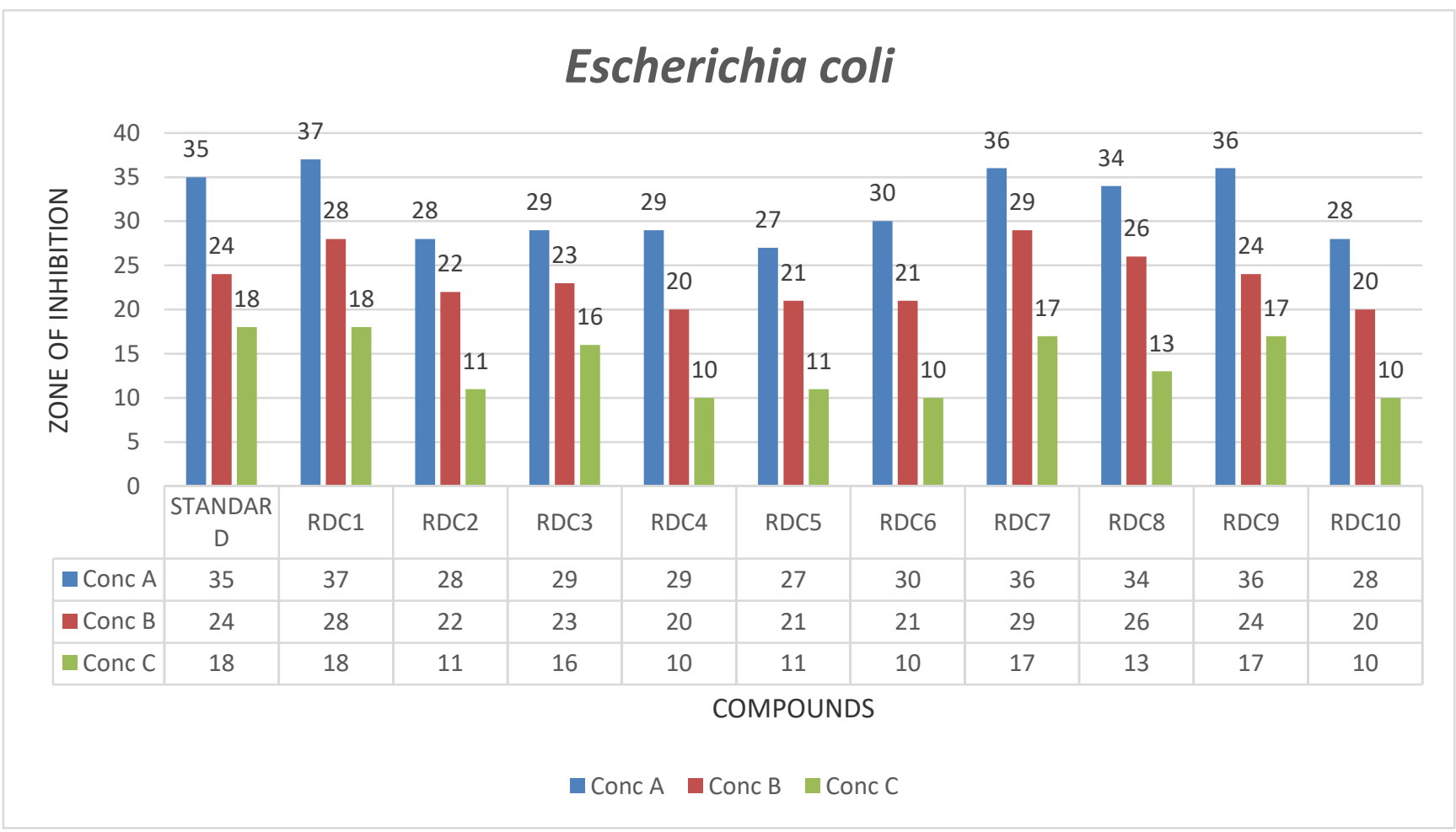

Graph 7. Comparative study of derived Schiff bases vs. doxycycline against Escherichia coli.

Derived Schiff bases RDC1, RDC7 and RDC9 showed zone of inhibition against Escherichia coli as compared to doxycycline.

\section{DISCUSSION}

\section{Gram +ve and Gram -ve Bacteria Compared with Standard Drug Doxycycline}

The Schiff bases synthesized by simple one-step reaction. All the ligands synthesized by simple condensation reaction, characterized and confirmed by using different techniques of spectroscopy i.e. FTIR and NMR.

Antibacterial activity of all the derived Schiff bases ligands performed against both gram +ve and gram ve bacteria and compared with standard drug doxycycline from which all of these Schiff base ligands derived.

D2, D4 and D10 show comparatively high activity against Bacillus megaterium as compared to doxycycline.

Derived Schiff bases D2, D4 and D10 are more active against Bacillus subtilis as compared to doxycycline.

Derived Schiff bases D2, D3, D4, D5, D6, D7 and D8 show high activity against Stenotrophomonas maltophilia as compared to doxycycline.
Derived Schiff bases D1, D2, D5, D6, and D7 show good Staphylococcus aureus as compare to doxycycline.

Derived Schiff bases D1, D2, D5, D6, D7 and D8 show maximum against Micrococcus luteus as compare to doxycycline.

Most derived Schiff bases D1, D2, D3, D5, D6, D7, D8, D9 and D10 show maximum zone of inhibition against Serratia marcescens as compared to doxycycline.

Derived Schiff bases D1, D7 and D9 showed zone of inhibition against Escherichia coli as compared to doxycycline.

Almost all the derived Schiff bases show moderate to maximum antibacterial activity against all the studied strains but most of the derived compounds are more active against gram-negative strains Stenotrophomonas maltophilia and Serratia marcescens. 


\section{CONCLUSION}

Antibacterial resistance against antibiotics is major problem in all over the world. We have developed resistance against many broad-spectrum antibiotics. To overcome this resistance against we need new antibiotics discovery or derived new compounds from pre-existing antibiotics. These derived compounds show activity against resistant bacteria. Doxycycline is among broad-spectrum tetracycline. The Schiff bases derived from doxycycline show significantly highly active against gram negative bacteria as compared to doxycycline. In future further study on these derived compounds will help in market new derivative of doxycycline, which will have more broadspectrum activity than doxycycline.

\section{REFERENCES}

1. Gold V, Loening $K$, McNaught A, Shemi P. lupac compendium of chemical terminology. Blackwell Science, Oxford. 1997.

2. Schiff H. Mittheilungen aus dem Universitätslaboratorium in Pisa: eine neue Reiheorganischer Basen. Eur J Org Chem. 1864; 131(1):118-9.

3. Tidwell TT. Hugo (Ugo) Schiff, Schiff bases, and a century of $\beta$-lactam synthesis. Angew Chem Int Ed Engl. 2008;47(6):1016-20.

4. Staudinger $H$. Zur Kenntniss der Ketene. Diphenylketen. Eur J Org Chem. 1907; 356(1-2):51123.

5. Hine J, Yeh CY. Equilibrium in formation and conformational isomerization of imines derived from isobutyraldehyde and saturated aliphatic primary amines. J Am Chem Soc. 1967; 89(11):2669-76.

6. Menger FM, Zana R, Lindman B. Portraying the structure of micelles. J Chem Educ. 1998; 75(1):115.

7. Shapiro HK. Carbonyl-trapping therapeutic strategies. Am J Therapeut. 1998; 5(5):323-54.

8. Pandeya SN, Sriram D, Nath G, De Clercq E. Synthesis and antimicrobial activity of Schiff and Mannich bases of isatin and its derivatives with pyrimidine. Farmaco. 1999; 54(9):624-8.

9. Shivarama Holla B, Veerendra B, Shivananda MK, Poojary B. Synthesis characterization and anticancer activity studies on some Mannich bases derived from 1,2,4-triazoles. Eur J Med Chem. 2003; 38(7-8):759-67.

10. Nirmal R, Meenakshi K, Shanmugapandiyan $P$, Prakash CR. Synthesis Pharmacological Evaluation of Novel Schiff Base Analogues of 3-(4-amino)
Phenylimino) 5-fluoroindolin-2-one. J Young Pharm. 2010; 2(2):162-8.

11. Verma M, Pandeya SN, Singh KN, Stables JP. Anticonvulsant activity of Schiff bases of isatin derivatives. Acta Pharmaceutica. 2004; 54(1):49-56.

12. Mathew B, Vakketh SS, Kumar SS. Synthesis, molecular properties and anthelmintic activity of some schiff bases of 1, 3, 4 thiadiazole derivatives. Der Pharma Chemica. 2010; 2(5):337-43.

13. Brodowska K, Sykuła A, Garribba E, ŁodygaChruścińska E, Sójka M. Naringenin Schiff base: antioxidant activity, acid-base profile, and interactions with DNA. Transition Metal Chem. 2016; 41(2):179-89.

14. Qin W, Long S, Panunzio M, Biondi S. Schiff bases: A short survey on an evergreen chemistry tool. Molecules. 2013; 18(10):12264-89.

15. Hussain Z, Khalaf M, Adil H, Zageer D, Hassan F, Mohammed S, et al. Metal Complexes of Schiff's Bases Containing Sulfonamides Nucleus: A Review. Res J Pharm Biol Chem Sci. 2016; 7(5):1008-25.

16. Cozzi PG. Metal-Salen Schiff base complexes in catalysis: practical aspects. Chem Soc Rev. 2004; 33(7):410-21.

17. Singh K, Barwa MS, Tyagi P. Synthesis and characterization of cobalt (II), nickel (II), copper (II) and zinc (II) complexes with Schiff base derived from 4-amino-3-mercapto-6-methyl-5-oxo-1, 2, 4-triazine. Eur J Med Chem. 2007; 42(3):394-402.

18. Nelson ML, Levy SB. The history of the tetracyclines. Ann NY Acad Sci. 2011; 1241(1):17-32.

19. Alam MJ, Mousumi SJ, Rana R, Islam MS, Akter MS, Juliana FM, et al. Ceftriaxone Resistance Patterns of Uropathogens Isolated from Urinary Tract Infection Patients in Selected Areas of Dhaka City, Bangladesh. IOSR J Nurs Health Sci. 2017; 6(5):2834.

20. Hilal-Dandan R, Brunton L. Goodman and Gilman Manual of Pharmacology and Therapeutics, 2. McGraw Hill Professional, Philadelphia; 2013.

21. Sweet RL, Schachter J, Landers DV, Ohm-Smith M, Robbie MO. Treatment of hospitalized patients with acute pelvic inflammatory disease: comparison of cefotetan plus doxycycline and cefoxitin plus doxycycline. Am J Obstet Gynecol. 1988; 158(3):73641.

22. Määttä $M$, Kari $O$, Tervahartiala $T$, Peltonen $S$, Kari $M$, Saari $M$, et al. Tear fluid levels of MMP-8 are elevated in ocular rosacea-treatment effect of oral doxycycline. Graefes Arch Clin Exp Ophthalmol. 2006; 244(8):957-62. 